\section{TATRA \\ MOUNTaiNS \\ Mathematical Publications}

DOI: $10.2478 / \mathrm{tmmp}-2021-0023$

Tatra Mt. Math. Publ. 79 (2021), 119-134

\title{
OSCILLATORY BEHAVIOUR OF SECOND-ORDER NONLINEAR DIFFERENTIAL EQUATIONS WITH MIXED NEUTRAL TERMS
}

\author{
Said R. Grace ${ }^{1}$ - John R. Graef ${ }^{2}$ - Tongxing LI $^{3}$ - Ercan TunÇ ${ }^{4}$ \\ ${ }^{1}$ Cairo University, Orman, EGYPT \\ ${ }^{2}$ University of Tennessee at Chattanooga, Chattanooga, TN, USA \\ ${ }^{3}$ Shandong University, Shandong, CHINA \\ ${ }^{4}$ Gaziosmanpasa University, Tokat, TURKEY
}

\begin{abstract}
The authors examine the oscillation of second-order nonlinear differential equations with mixed nonlinear neutral terms. They present new oscillation criteria that improve, extend, and simplify existing ones in the literature. The results are illustrated by some examples.
\end{abstract}

\section{Introduction}

This paper is concerned with the oscillatory behaviour of solutions of the second-order nonlinear differential equations with mixed neutral terms of the form

$$
\left(a(t)\left(y^{\prime}(t)\right)^{\alpha}\right)^{\prime}+q(t) x^{\gamma}(\tau(t))+c(t) x^{\mu}(\omega(t))=0
$$

and

$$
\left(a(t)\left(y^{\prime}(t)\right)^{\alpha}\right)^{\prime}=q(t) x^{\gamma}(\tau(t))+c(t) x^{\mu}(\omega(t)),
$$

where $y(t)=x(t)+p_{1}(t) x^{\beta}(\sigma(t))-p_{2}(t) x^{\delta}(\sigma(t))$ and $t \geq t_{0}>0$. Throughout this paper, we always assume that the following conditions are satisfied:

(i) $\alpha, \beta, \gamma, \mu$, and $\delta$ are the ratios of odd positive integers with $0<\beta<1$ and $\delta>1$

(C) 2021 Mathematical Institute, Slovak Academy of Sciences.

2010 Mathematics Subject Classification: 34C10, 34C15, 34K11, 34K40.

Keyw ords: oscillation, second order, neutral differential equations, mixed neutral term.

(ㄷ) $(1) \Theta$ Licensed under the Creative Commons BY-NC-ND 4.0 International Public License. 
(ii) $a:\left[t_{0}, \infty\right) \rightarrow(0, \infty)$ is a continuous function such that

$$
A\left(t, t_{0}\right):=\int_{t_{0}}^{t} a^{-1 / \alpha}(s) \mathrm{d} s \rightarrow \infty \text { as } t \rightarrow \infty ;
$$

(iii) $p_{1}, p_{2}, q, c:\left[t_{0}, \infty\right) \rightarrow(0, \infty)$ are continuous functions;

(iv) $\tau, \sigma, \omega:\left[t_{0}, \infty\right) \rightarrow \mathbb{R}$ are continuous functions such that $\tau(t) \leq t, \sigma(t) \leq t$, $\omega(t) \geq t$, and $\lim _{t \rightarrow \infty} \tau(t)=\lim _{t \rightarrow \infty} \sigma(t)=\lim _{t \rightarrow \infty} \omega(t)=\infty$.

By a solution of equation (11) (resp. (2) ), we mean a function $x \in C\left(\left[t_{x}, \infty\right), \mathbb{R}\right.$ ) for some $t_{x} \geq t_{0}$ such that $y \in C^{1}\left(\left[t_{x}, \infty\right), \mathbb{R}\right), a\left(y^{\prime}\right)^{\alpha} \in C^{1}\left(\left[t_{x}, \infty\right), \mathbb{R}\right)$, and satisfies (11) (respectively (2)) on $\left[t_{x}, \infty\right)$. We only consider those solutions of (11) (respectively (2)) that exist on some half-line $\left[t_{x}, \infty\right)$ and satisfy the condition

$$
\sup \left\{|x(t)|: T_{1} \leq t<\infty\right\}>0 \text { for any } T_{1} \geq t_{x} .
$$

Moreover, we tacitly assume that (10) (respectively (2)) possesses such solutions. Such a solution $x(t)$ of either (1) or (2) is said to be oscillatory if it has arbitrarily large zeros on $\left[t_{x}, \infty\right)$, i.e., for any $t_{1} \in\left[t_{x}, \infty\right)$ there exists $t_{2} \geq t_{1}$ such that $x\left(t_{2}\right)=0$; otherwise it is called nonoscillatory, i.e., if it is eventually positive or eventually negative. Equation (11) or (2) is said to be oscillatory if all its solutions are oscillatory.

In recent years, there has been much research activity concerning the oscillation and nonoscillation of solutions of various differential equations, and we refer the reader to the monographs [2, 3, and the papers [1, 4, 10, 14, 22 and the references cited therein. With respect to neutral differential equations, the qualitative study of such equations has, besides its theoretical interest, significant practical importance. This is due to the fact that neutral differential equations arise in various phenomena including problems concerning electric networks containing lossless transmission lines (as in high-speed computers where such lines are used to interconnect switching circuits), in the study of vibrating masses attached to an elastic bar, and in the solution of vibrational problems with time delays. We refer the reader to Hale's monograph [11] for further applications in science and technology.

In reviewing the literature, it becomes apparent that results on the oscillatory behaviour of the second-order differential equations with a single sublinear neutral term are relatively scarce. For an important initial contribution for such equations, we may refer to [1,6]. By using Riccati type transformations, in [1, the authors obtain some oscillation results for (11) in the case where $\alpha=\gamma=1$, $p_{2}(t)=0$, and $c(t)=0$. On the other hand, the authors in [6] established some new results for the case where $\alpha=1, p_{2}(t)=0$, and $c(t)=0$. 
However, to the best of our knowledge, there are few results dealing with the oscillation of mixed neutral differential equations with both sublinear and superlinear neutral terms; for example, see [8], where an equation with nonnegative neutral terms was considered. The aim of the present paper is to initiate the study of the oscillatory behaviour of (11) and to provide new results that extend, generalize, and simplify existing ones in the literature, and to analyse the oscillatory and asymptotic behaviour of solutions of the corresponding equation (2) with mixed neutral terms and again under condition (3) with $\beta<1$ and $\delta>1$.

\section{Oscillation of equation (1)}

We begin with the following lemma that is essential in the proofs of our theorems.

LEMma 2.1 ([12]). If $X$ and $Y$ are nonnegative, then

$$
X^{\lambda}+(\lambda-1) Y^{\lambda}-\lambda X Y^{\lambda-1} \geq 0 \quad \text { for } \quad \lambda>1
$$

and

$$
X^{\lambda}-(1-\lambda) Y^{\lambda}-\lambda X Y^{\lambda-1} \leq 0 \quad \text { for } \quad 0<\lambda<1,
$$

where equality holds if and only if $X=Y$.

For notational purposes, we let

$$
A(v, u):=\int_{u}^{v} a^{-1 / \alpha}(s) \mathrm{d} s,
$$

and for any function $p \in C\left(\left[t_{0}, \infty\right), \mathbb{R}\right)$, we set

$$
\begin{gathered}
g_{1}(t):=(\delta-1) \delta^{\delta /(1-\delta)} p^{\delta /(\delta-1)}(t) p_{2}^{1 /(1-\delta)}(t) \\
g_{2}(t):=(1-\beta) \beta^{\beta /(1-\beta)} p^{\beta /(\beta-1)}(t) p_{1}^{1 /(1-\beta)}(t), \\
Q(t):=\frac{q(t)}{\left(p_{2}\left(h_{1}(t)\right)\right)^{\gamma / \delta}}
\end{gathered}
$$

and

$$
C(t):=\frac{c(t)}{\left(p_{2}\left(h_{2}(t)\right)\right)^{\mu / \delta}},
$$

where $h_{1}(t)=\sigma^{-1}(\tau(t)) \leq t$ with $\lim _{t \rightarrow \infty} h_{1}(t)=\infty, h_{2}(t)=\sigma^{-1}(\omega(t))$ with $\lim _{t \rightarrow \infty} h_{2}(t)=\infty, h_{2}^{\prime}(t)>0$, and $\sigma^{-1}$ is the inverse function of $\sigma$. 


\section{SAID R. GRACE-JOHN R. GRAEF-TONGXING LI —ERCAN TUNC}

Our first main result is contained in the following theorem for Eq. (11).

TheOREM 2.2. Let conditions (i)-(iv) and (3) hold. Assume that there exist functions $p \in C\left(\left[t_{0}, \infty\right), \mathbb{R}\right), \varphi \in C\left(\left[t_{0}, \infty\right), \mathbb{R}\right)$, and a nondecreasing function $\xi \in C\left(\left[t_{0}, \infty\right), \mathbb{R}\right)$ such that

and

$$
\begin{gathered}
\lim _{t \rightarrow \infty}\left[g_{1}(t)+g_{2}(t)\right]=0, \\
\varphi(t) \leq t \quad \text { and } \quad \rho(t):=h_{2}(\varphi(t)) \geq t
\end{gathered}
$$

If there is a $\kappa_{0} \in(0,1)$ such that the first-order delay differential inequality

$$
Z^{\prime}(t)+\kappa_{0}^{\gamma} q(t) A^{\gamma}\left(\tau(t), t_{0}\right) Z^{\gamma / \alpha}(\tau(t)) \leq 0,
$$

the first-order delay differential inequality

$$
W^{\prime}(t)+Q(t) A^{\gamma / \delta}\left(\xi(t), h_{1}(t)\right) W^{\gamma / \alpha \delta}(\xi(t)) \leq 0,
$$

and the first-order advanced differential inequality

$$
z^{\prime}(t)-\left(\frac{1}{a(t)} \int_{\varphi(t)}^{t} C(s) \mathrm{d} s\right)^{1 / \alpha} z^{\mu / \alpha \delta}(\rho(t)) \geq 0
$$

have no positive solutions, then equation (1) is oscillatory.

P r o of. Let $x(t)$ be a nonoscillatory solution to the equation (11), say $x(t)>0$, $x(\sigma(t))>0, x(\tau(t))>0$ and $x(\omega(t))>0$ for $t \geq t_{1}$ for some $t_{1} \geq t_{0}$. The proof if $x(t)$ is eventually negative is similar, so we omit the details of that case here as well as in the remaining proofs in this paper. Then, it follows from (11) that

$$
\left(a(t)\left(y^{\prime}(t)\right)^{\alpha}\right)^{\prime}=-q(t) x^{\gamma}(\tau(t))-c(t) x^{\mu}(\omega(t)) \leq-q(t) x^{\gamma}(\tau(t))<0,
$$

for $t \geq t_{1}$, and hence $a(t)\left(y^{\prime}(t)\right)^{\alpha}$ is decreasing and eventually does not change its sign, say on $\left[t_{2}, \infty\right)$ for some $t_{2} \geq t_{1}$. Therefore, $y^{\prime}(t)$ eventually has a fixed sign on $\left[t_{2}, \infty\right)$, and so we shall distinguish the following four cases:

$$
\begin{aligned}
& \text { (I) } y(t)>0 \text { and } y^{\prime}(t)<0 \text {, } \\
& \text { (II) } y(t)>0 \text { and } y^{\prime}(t)>0 \text {, } \\
& \text { (III) } y(t)<0 \text { and } y^{\prime}(t)>0 \text {, } \\
& \text { (IV) } y(t)<0 \text { and } y^{\prime}(t)<0 \text {. }
\end{aligned}
$$

First, we consider case (I). Since $y^{\prime}(t)<0$ and $a(t)\left(y^{\prime}(t)\right)^{\alpha}$ is decreasing for $t \geq t_{2}$, we see that, for $c_{1}>0$,

$$
a(t)\left(y^{\prime}(t)\right)^{\alpha} \leq a\left(t_{2}\right)\left(y^{\prime}\left(t_{2}\right)\right)^{\alpha}:=-c_{1}<0 .
$$

Integrating the last inequality from $t_{2}$ to $t$ and taking (3) into account, we conclude that $\lim _{t \rightarrow \infty} y(t)=-\infty$, which contradicts the fact that $y(t)$ is eventually positive. 


\section{OSCILLATORY BEHAVIOUR OF SECOND-ORDER DIFFERENTIAL EQUATIONS}

Next, we consider case (II). From the definition of $y(t)$, we have

$$
x(t)=y(t)-\left[p(t) x(\sigma(t))-p_{2}(t) x^{\delta}(\sigma(t))\right]-\left[p_{1}(t) x^{\beta}(\sigma(t))-p(t) x(\sigma(t))\right] .
$$

Applying (44) to $\left[p(t) x(\sigma(t))-p_{2}(t) x^{\delta}(\sigma(t))\right]$ with

$$
\lambda=\delta>1, X=p_{2}^{1 / \delta}(t) x(\sigma(t)) \quad \text { and } \quad Y=\left(\frac{1}{\delta} p(t) p_{2}^{-1 / \delta}(t)\right)^{1 /(\delta-1)},
$$

we see that

$$
\left[p(t) x(\sigma(t))-p_{2}(t) x^{\delta}(\sigma(t))\right] \leq(\delta-1) \delta^{\delta /(1-\delta)} p^{\delta /(\delta-1)}(t) p_{2}^{1 /(1-\delta)}(t):=g_{1}(t) .
$$

Applying (5) to $\left[p_{1}(t) x^{\beta}(\sigma(t))-p(t) x(\sigma(t))\right]$ with

$$
\lambda=\beta<1, X=p_{1}^{1 / \beta}(t) x(\sigma(t)) \quad \text { and } \quad Y=\left(\frac{1}{\beta} p(t) p_{1}^{-1 / \beta}(t)\right)^{1 /(\beta-1)},
$$

we see that

$$
\left[p_{1}(t) x^{\beta}(\sigma(t))-p(t) x(\sigma(t))\right] \leq(1-\beta) \beta^{\beta /(1-\beta)} p^{\beta /(\beta-1)}(t) p_{1}^{1 /(1-\beta)}(t):=g_{2}(t) .
$$

Using (14) and (15) in (13) gives

$$
x(t) \geq\left[1-\frac{g_{1}(t)+g_{2}(t)}{y(t)}\right] y(t) \text { for } t \geq t_{2} .
$$

Since $y(t)>0$ and $y^{\prime}(t)>0$ on $\left[t_{2}, \infty\right)$, there exist $t_{3} \geq t_{2}$ and a constant $c_{2}>0$ such that $y(t) \geq c_{2}$ for $t \geq t_{3}$, and so, inequality (16) can be written as

$$
x(t) \geq\left[1-\frac{g_{1}(t)+g_{2}(t)}{c_{2}}\right] y(t) \text { for } t \geq t_{3} .
$$

Now, in view of (6) , for any $\kappa \in(0,1)$ there exists $t_{\kappa} \geq t_{3}$ such that

$$
x(t) \geq \kappa y(t) \text { for } t \geq t_{\kappa} .
$$

Fix $\kappa \in(0,1)$ and choose $t_{\kappa}$ by (18) . Since $\lim _{t \rightarrow \infty} \tau(t)=\infty$, we can choose $t_{5} \geq t_{\kappa}$ such that $\tau(t) \geq t_{\kappa}$ for all $t \geq t_{5}$. Thus, from (18) we have

$$
x(\tau(t)) \geq \kappa y(\tau(t)) \text { for } t \geq t_{5} .
$$

Using (19) in (12) yields

$$
\left(a(t)\left(y^{\prime}(t)\right)^{\alpha}\right)^{\prime}+\kappa^{\gamma} q(t) y^{\gamma}(\tau(t)) \leq 0 \quad \text { for } t \geq t_{5} .
$$

Since $y(t)$ is positive and $a(t)\left(y^{\prime}(t)\right)^{\alpha}$ is decreasing for $t \geq t_{5}$, we see that

$$
\begin{aligned}
y(t) & \geq \int_{t_{5}}^{t} a^{-1 / \alpha}(s)\left(a^{1 / \alpha}(s) y^{\prime}(s)\right) \mathrm{d} s \\
& \geq A\left(t, t_{5}\right)\left(a(t)\left(y^{\prime}(t)\right)^{\alpha}\right)^{1 / \alpha} .
\end{aligned}
$$


Letting $Z(t)=a(t)\left(y^{\prime}(t)\right)^{\alpha}>0$, inequality (21) takes the form

$$
y(t) \geq A\left(t, t_{5}\right) Z^{1 / \alpha}(t) .
$$

Using (22) in (20), we see that $Z$ is a positive solution of the inequality

$$
Z^{\prime}(t)+\kappa^{\gamma} q(t) A^{\gamma}\left(\tau(t), t_{5}\right) Z^{\gamma / \alpha}(\tau(t)) \leq 0,
$$

i.e., inequality (9) has a positive solution, which contradicts our assumption.

Next, we consider the cases where $y(t)<0$ for $t \geq t_{2}$, i.e., cases (III) and (IV). Letting $z(t)=-y(t)$, then from the definition of $y(t)$, we see that

$$
z(t)=-y(t)=-x(t)-p_{1}(t) x^{\beta}(\sigma(t))+p_{2}(t) x^{\delta}(\sigma(t)) \leq p_{2}(t) x^{\delta}(\sigma(t)),
$$

from which, we obtain

or

$$
x(\sigma(t)) \geq\left(\frac{z(t)}{p_{2}(t)}\right)^{1 / \delta}
$$

$$
x(t) \geq\left(\frac{z\left(\sigma^{-1}(t)\right)}{p_{2}\left(\sigma^{-1}(t)\right)}\right)^{1 / \delta} \quad \text { for } t \geq t_{2} .
$$

In case (III), we have

$$
z(t)=-y(t)>0 \quad \text { for } t \geq t_{2}, \quad \text { so } \quad z^{\prime}(t)=-y^{\prime}(t)<0 \quad \text { for } t \geq t_{2} .
$$

Now, it follows from (12) that

$$
\left(a(t)\left(z^{\prime}(t)\right)^{\alpha}\right)^{\prime} \geq q(t) x^{\gamma}(\tau(t)) \text { for } t \geq t_{2} .
$$

Using (24) in (25) gives

$$
\left(a(t)\left(z^{\prime}(t)\right)^{\alpha}\right)^{\prime} \geq Q(t) z^{\gamma / \delta}\left(h_{1}(t)\right) \text { for } t \geq t_{3},
$$

where $\tau(t) \geq t_{2}$ for $t \geq t_{3}$ for some $t_{3} \geq t_{2}$. Now, for $t_{3} \leq u \leq v$, we may write

$$
z(u)-z(v)=-\int_{u}^{v} a^{-1 / \alpha}(s)\left(a(s)\left(z^{\prime}(s)\right)^{\alpha}\right)^{1 / \alpha} \mathrm{d} s \geq A(v, u)\left(-a^{1 / \alpha}(v) z^{\prime}(v)\right) .
$$

Letting $u=h_{1}(t)$ and $v(t)=\xi(t)$ in the last inequality, we see that

$$
z\left(h_{1}(t)\right) \geq A\left(\xi(t), h_{1}(t)\right)\left(-a^{1 / \alpha}(\xi(t)) z^{\prime}(\xi(t))\right) .
$$

Using (27) in (26) gives

$$
\left(a(t)\left(z^{\prime}(t)\right)^{\alpha}\right)^{\prime} \geq Q(t)\left[A\left(\xi(t), h_{1}(t)\right)\left(-a^{1 / \alpha}(\xi(t)) z^{\prime}(\xi(t))\right)\right]^{\gamma / \delta} \quad \text { for } t \geq t_{3} .
$$

Letting $W(t)=a(t)\left(-z^{\prime}(t)\right)^{\alpha}>0$, we see that $W(t)$ is a positive solution of the first-order delay differential inequality

$$
W^{\prime}(t)+Q(t) A^{\gamma / \delta}\left(\xi(t), h_{1}(t)\right) W^{\gamma / \alpha \delta}(\xi(t)) \leq 0,
$$

which contradicts the assumption that (10) has no positive solutions. 


\section{OSCILLATORY BEHAVIOUR OF SECOND-ORDER DIFFERENTIAL EQUATIONS}

Finally, we consider case (IV). Since $y(t)<0$ for $t \geq t_{2}$, as in the above, letting $z(t)=-y(t)>0$ for $t \geq t_{2}$, we again arrive at (24). Using (24) in (11), we obtain

$$
\begin{aligned}
\left(a(t)\left(z^{\prime}(t)\right)^{\alpha}\right)^{\prime} & =q(t) x^{\gamma}(\tau(t))+c(t) x^{\mu}(\omega(t)) \\
& \geq Q(t) z^{\gamma / \delta}\left(h_{1}(t)\right)+C(t) z^{\mu / \delta}\left(h_{2}(t)\right) \\
& \geq C(t) z^{\mu / \delta}\left(h_{2}(t)\right) .
\end{aligned}
$$

Integrating (30) from $\varphi(t)$ to $t$, we see that $z(t)$ is a positive solution of the first-order advanced inequality

$$
z^{\prime}(t) \geq\left(\frac{1}{a(t)} \int_{\varphi(t)}^{t} C(s) \mathrm{d} s\right)^{1 / \alpha} z^{\mu / \alpha \delta}(\rho(t)),
$$

which contradicts the assumption on inequality (11), and completes the proof of the theorem.

Corollary 2.3. Let conditions (i)-(iv) and (3) hold. Assume that there exist functions $p \in C\left(\left[t_{0}, \infty\right), \mathbb{R}\right), \varphi \in C\left(\left[t_{0}, \infty\right), \mathbb{R}\right)$, and nondecreasing function $\xi \in$ $C\left(\left[t_{0}, \infty\right), \mathbb{R}\right)$ such that (6) (6) are satisfied. If

$$
\begin{aligned}
\int_{t_{0}}^{\infty} q(s) A^{\gamma}\left(\tau(s), t_{0}\right) \mathrm{d} s & =\infty \quad \text { if } \quad \gamma<\alpha, \\
\int_{t_{0}}^{\infty} Q(s) A^{\gamma / \delta}\left(\xi(s), h_{1}(s)\right) \mathrm{d} s & =\infty \quad \text { if } \quad \gamma<\alpha \delta,
\end{aligned}
$$

and

$$
\int_{t_{0}}^{\infty}\left(\frac{1}{a(u)} \int_{\varphi(u)}^{u} C(s) \mathrm{d} s\right)^{1 / \alpha} \mathrm{d} u=\infty \quad \text { if } \quad \mu>\alpha \delta,
$$

then equation (11) is oscillatory.

Pro of. Let $x(t)$ be a nonoscillatory solution to the equation (1), say $x(t)>0$ $x(\sigma(t))>0, x(\tau(t))>0$ and $x(\omega(t))>0$ for $t \geq t_{1}$ for some $t_{1} \geq t_{0}$. Proceeding as in the proof of Theorem 2.2. we again arrive at (23) for $t \geq t_{5}$, (29) for $t \geq t_{3}$, and (31) for $t \geq t_{2}$, respectively. Using the fact that $Z(t)=$ $a(t)\left(y^{\prime}(t)\right)^{\alpha}$ is positive and decreasing, and noting that $\tau(t) \leq t$, we have

$$
Z(\tau(t)) \geq Z(t),
$$


and so, inequality (23) can be written as

$$
Z^{\prime}(t)+\kappa^{\gamma} q(t) A^{\gamma}\left(\tau(t), t_{5}\right) Z^{\gamma / \alpha}(t) \leq 0
$$

or

$$
\frac{Z^{\prime}(t)}{Z^{\gamma / \alpha}(t)}+\kappa^{\gamma} q(t) A^{\gamma}\left(\tau(t), t_{5}\right) \leq 0 \quad \text { for } t \geq t_{5} .
$$

An integration of (35) from $t_{5}$ to $\infty$ gives

$$
\int_{t_{5}}^{\infty} q(s) A^{\gamma}\left(\tau(s), t_{5}\right) \mathrm{d} s \leq \frac{1}{\kappa^{\gamma}} \frac{Z^{1-\frac{\gamma}{\alpha}}\left(t_{5}\right)}{1-\frac{\gamma}{\alpha}}<\infty,
$$

which contradicts (32). Using similar arguments, the remainder of proof follows from the facts that $\xi(t) \leq t, \rho(t) \geq t$, and inequalities (29) and (31); we omit the details.

\section{Oscillation of equation (2)}

In this section we examine the behaviour of solutions of equation (2).

Theorem 3.1. Let conditions (i)-(iv) and (3) hold. Assume that there exist functions $p \in C\left(\left[t_{0}, \infty\right),(0, \infty)\right)$ and $\eta \in C\left(\left[t_{0}, \infty\right), \mathbb{R}\right)$ such that (66) holds,

$$
\eta(t) \leq t, \quad \omega^{\prime}(t) \geq 0 \quad \text { and } \quad \pi(t):=\omega(\eta(t)) \geq t,
$$

and either

$$
\int_{t_{0}}^{\infty} q(s) \mathrm{d} s=\infty \quad \text { or } \int_{t_{0}}^{\infty}\left(\frac{1}{a(u)} \int_{u}^{\infty} q(s) \mathrm{d} s\right)^{1 / \alpha} \mathrm{d} u=\infty,
$$

are satisfied. In addition, if there is a $\kappa_{0} \in(0,1)$ such that the first-order advanced differential inequality

$$
z^{\prime}(t)-\kappa_{0}^{\mu / \alpha}\left(\frac{1}{a(t)} \int_{\eta(t)}^{t} c(s) \mathrm{d} s\right)^{1 / \alpha} z^{\mu / \alpha}(\pi(t)) \geq 0,
$$

and the first-order delay differential inequality

$$
Z^{\prime}(t)+Q(t) A^{\gamma / \delta}\left(h_{1}(t), t_{0}\right) Z^{\gamma / \alpha \delta}\left(h_{1}(t)\right) \leq 0,
$$

have no positive solutions. If $x(t)$ is a solution to the equation (2), then either $x(t)$ is oscillatory or $\liminf _{t \rightarrow \infty}|x(t)|=0$. 
P r o of. Let $x(t)$ be a nonoscillatory solution to the equation (2), say $x(t)>0$, $x(\sigma(t))>0, x(\tau(t))>0$, and $x(\omega(t))>0$ for $t \geq t_{1}$, for some $t_{1} \geq t_{0}$. Then, it follows from (2) that

$$
\left(a(t)\left(y^{\prime}(t)\right)^{\alpha}\right)^{\prime}=q(t) x^{\gamma}(\tau(t))+c(t) x^{\mu}(\omega(t)) \geq q(t) x^{\gamma}(\tau(t))>0,
$$

for $t \geq t_{1}$, and hence $a(t)\left(y^{\prime}(t)\right)^{\alpha}$ is increasing and eventually does not change its sign on $\left[t_{2}, \infty\right)$ for some $t_{2} \geq t_{1}$. Therefore, $y^{\prime}(t)$ eventually has a fixed sign on $\left[t_{2}, \infty\right)$, and so we shall distinguish the following four cases:

$$
\begin{aligned}
& \text { (I) } y(t)>0 \text { and } y^{\prime}(t)<0, \quad \text { (II) } y(t)>0 \quad \text { and } \quad y^{\prime}(t)>0 \text {, } \\
& \text { (III) } y(t)<0 \text { and } y^{\prime}(t)>0, \quad(\text { IV }) \quad y(t)<0 \quad \text { and } \quad y^{\prime}(t)<0 \text {. }
\end{aligned}
$$

First, we consider case (I): In this case, we claim that $\liminf _{t \rightarrow \infty} x(t)=0$. To prove this, we assume that there exists a constant $b>0$ such that $x(t)>b$. Using this in (40), we see that

$$
\left(a(t)\left(y^{\prime}(t)\right)^{\alpha}\right)^{\prime} \geq b^{\gamma} q(t) .
$$

Integrating (41) from $t_{2}$ to $\infty$, we see that

$$
\int_{t_{2}}^{\infty} q(s) \mathrm{d} s \leq \frac{-a\left(t_{2}\right)\left(y^{\prime}\left(t_{2}\right)\right)^{\alpha}}{b^{\gamma}}<\infty,
$$

which contradicts the first part of (37). If we integrate (41) from $t$ to $u$ and letting $u \rightarrow \infty$, we obtain

$$
-y^{\prime}(t) \geq\left[b^{\gamma} \frac{1}{a(t)} \int_{t}^{\infty} q(s) \mathrm{d} s\right]^{1 / \alpha} .
$$

Integrating (42) from $t_{2}$ to $\infty$ and using the second part of (37), we again arrive at the desired contradiction.

Next, we consider case (II). Proceeding exactly as in the proof of Theorem 2.2. we again arrive at (18). Using (18) in (2), we see that

$$
\left(a(t)\left(y^{\prime}(t)\right)^{\alpha}\right)^{\prime} \geq \kappa^{\mu} c(t) y^{\mu}(\omega(t)) \text { for } t \geq t_{5} .
$$

Integrating (43) from $\eta(t)$ to $t$, we see that $y(t)$ is a positive solution of the first-order advanced differential inequality

$$
y^{\prime}(t) \geq \kappa^{\mu / \alpha}\left(\frac{1}{a(t)} \int_{\eta(t)}^{t} c(s) \mathrm{d} s\right)^{1 / \alpha} y^{\mu / \alpha}(\pi(t)),
$$

which contradicts the assumption on inequality (38). 


\section{SAID R. GRACE-JOHN R. GRAEF-TONGXING LI —ERCAN TUNÇ}

In case (III), since $y(t)<0$ for $t \geq t_{2}$, as in the proof of case (III) in Theorem 2.2, we let $z(t)=-y(t)>0$ and see that (24) holds. Using (24) in (40), we obtain

$$
\left(a(t)\left(z^{\prime}(t)\right)^{\alpha}\right)^{\prime}+Q(t) z^{\gamma / \delta}\left(h_{1}(t)\right) \leq 0 \text { for } t \geq t_{2} .
$$

Using the fact that $z^{\prime}(t)=-y^{\prime}(t)<0$ and $a(t)\left(z^{\prime}(t)\right)^{\alpha}$ is decreasing, and taking into account (3), as in the proof of case (I) in Theorem 2.2, we contradicts the fact that $z(t)$ is eventually positive.

Finally, for case (IV), letting $z(t)=-y(t)>0$, we again arrive at (45). Using the fact that $z(t)>0$ and $a(t)\left(z^{\prime}(t)\right)^{\alpha}$ is decreasing, we see that

$$
z(t) \geq \int_{t_{2}}^{t} a^{-1 / \alpha}(s)\left(a(s)\left(z^{\prime}(s)\right)^{\alpha}\right)^{1 / \alpha} \mathrm{d} s \geq A\left(t, t_{2}\right)\left(a^{1 / \alpha}(t) z^{\prime}(t)\right) .
$$

Letting $Z(t)=a(t)\left(z^{\prime}(t)\right)^{\alpha}>0$, in (46) yields

$$
z(t) \geq A\left(t, t_{2}\right) Z^{1 / \alpha}(t) .
$$

Substituting (47) into (45), we see that $Z(t)$ is a positive solution of the first-order delay differential inequality

$$
Z^{\prime}(t)+Q(t) A^{\gamma / \delta}\left(h_{1}(t), t_{2}\right) Z^{\gamma / \alpha \delta}\left(h_{1}(t)\right) \leq 0,
$$

which contradicts our assumption on inequality (39) and completes the proof.

It is well-known from [13] (see also [2, Lemma 2.2.9]) that if

$$
\liminf _{t \rightarrow \infty} \int_{\zeta(t)}^{t} R(s) \mathrm{d} s>\frac{1}{e},
$$

then the first-order delay differential inequality

$$
x^{\prime}(t)+R(t) x(\zeta(t)) \leq 0
$$

where $R, \zeta \in C\left(\left[t_{0}, \infty\right), \mathbb{R}\right)$ with $R(t) \geq 0, \zeta(t) \leq t$, and $\lim _{t \rightarrow \infty} \zeta(t)=\infty$, has no eventually positive solutions. If $\zeta(t) \geq t$, and $\zeta^{\prime}(t) \geq 0$, we have the following result (see [2, Lemma 2.2.10]): If

$$
\liminf _{t \rightarrow \infty} \int_{t}^{\zeta(t)} R(s) \mathrm{d} s>\frac{1}{e}
$$

then the first-order advanced differential inequality

$$
x^{\prime}(t)-R(t) x(\zeta(t)) \geq 0
$$

has no eventually positive solutions. 


\section{OSCILLATORY BEHAVIOUR OF SECOND-ORDER DIFFERENTIAL EQUATIONS}

Thus, from Theorem 3.1, we have the following oscillation result for equation (2).

Corollary 3.2. Let conditions (i)-(iv) and (3) hold. Assume that there exist functions $p \in C\left(\left[t_{0}, \infty\right), \mathbb{R}\right)$ and $\eta \in C\left(\left[t_{0}, \infty\right), \mathbb{R}\right)$ such that ([6) and (36) hold. If condition (37),

$$
\liminf _{t \rightarrow \infty} \int_{t}^{\pi(t)}\left(\frac{1}{a(u)} \int_{\eta(u)}^{u} c(s) \mathrm{d} s\right)^{1 / \alpha} \mathrm{d} u>\frac{1}{e} \quad \text { if } \mu=\alpha,
$$

and

$$
\liminf _{t \rightarrow \infty} \int_{h_{1}(t)}^{t} Q(s) A^{\gamma / \delta}\left(h_{1}(s), t_{0}\right) \mathrm{d} s>\frac{1}{e} \quad \text { if } \gamma=\alpha \delta,
$$

are satisfied, then a solution $x(t)$ of equation (2) is either oscillatory or satisfies $\liminf _{t \rightarrow \infty}|x(t)|=0$.

Pr o of. The proof is straightforward and we omit the details.

Corollary 3.3. Let conditions (i)-(iv) and (3) hold. Assume that there exist functions $p \in C\left(\left[t_{0}, \infty\right), \mathbb{R}\right)$ and $\eta \in C\left(\left[t_{0}, \infty\right), \mathbb{R}\right)$ such that (66) and (36) hold. If condition (37),

$$
\int_{t_{0}}^{\infty}\left(\frac{1}{a(u)} \int_{\eta(u)}^{u} c(s) \mathrm{d} s\right)^{1 / \alpha} \mathrm{d} u=\infty \quad \text { if } \mu>\alpha,
$$

and

$$
\int_{t_{0}}^{\infty} Q(s) A^{\gamma / \delta}\left(h_{1}(s), t_{0}\right) \mathrm{d} s=\infty \quad \text { if } \gamma<\alpha \delta,
$$

are satisfied, then a solution $x(t)$ of equation (2) is either oscillatory or satisfies $\liminf _{t \rightarrow \infty}|x(t)|=0$.

Proof. The proof is similar to the proof of Corollary 2.3, and hence we omit the details.

We conclude this paper with some examples to illustrate the above results and some suggestions for future research. 


\section{Examples}

EXAMPLE 1.

Consider the equation

$$
\left(t y^{\prime}(t)\right)^{\prime}+\left(1+t^{2}\right) x^{1 / 3}(t / 4)+(4 t)^{\mu / 3} x^{\mu}(2 t)=0, \quad t \geq 1,
$$

with

$$
y(t)=x(t)+\frac{1}{t} x^{1 / 3}(t / 2)-t x^{3}(t / 2) .
$$

Here

$$
\begin{aligned}
& \alpha=1, \quad \gamma=1 / 3, \quad \beta=1 / 3, \quad \delta=3, \quad \tau(t)=t / 4, \\
& \sigma(t)=t / 2, \quad \omega(t)=2 t, \quad a(t)=t, \quad q(t)=1+t^{2}, \quad c(t)=(4 t)^{\mu / 3}
\end{aligned}
$$

with $\mu>3$ is the ratio of positive odd integers,

$$
p_{1}(t)=1 / t \quad \text { and } \quad p_{2}(t)=t .
$$

Then, it is easy to see that conditions (i)-(iv) and (3) hold. Letting $p(t)=1$, we see that condition (6) holds. Letting $\xi(t)=2 t / 3$ and $\varphi(t)=t / 2$, we see that

$$
h_{1}(t)=\sigma^{-1}(\tau(t))=t / 2 \leq 2 t / 3,
$$

and

$$
\rho(t):=h_{2}(\varphi(t))=4 t>t,
$$

i.e, conditions (7) and (8) hold. Since

$$
A\left(t, t_{0}\right)=A(t, 1)=\int_{1}^{t} \frac{\mathrm{d} s}{s}=\ln t,
$$

we see that

$$
\begin{aligned}
\int_{t_{0}}^{\infty} q(s) A^{\gamma}\left(\tau(s), t_{0}\right) \mathrm{d} s & =\int_{1}^{\infty}\left(1+s^{2}\right)\left(\ln \frac{s}{4}\right)^{1 / 3} \mathrm{~d} s=\infty, \\
\int_{t_{0}}^{\infty} Q(s) A^{\gamma / \delta}\left(\xi(s), h_{1}(s)\right) \mathrm{d} s & =\int_{1}^{\infty} \frac{2^{1 / 9}\left(1+s^{2}\right)}{s^{1 / 9}}\left(\ln \frac{4}{3}\right)^{1 / 9} \mathrm{~d} s=\infty,
\end{aligned}
$$

and

$$
\int_{t_{0}}^{\infty}\left(\frac{1}{a(u)} \int_{\varphi(u)}^{u} C(s) \mathrm{d} s\right)^{1 / \alpha} \mathrm{d} u=\int_{1}^{\infty}\left(\frac{1}{u} \int_{u / 2}^{u} \mathrm{~d} s\right) \mathrm{d} u=\int_{1}^{\infty} \frac{1}{2} \mathrm{~d} u=\infty,
$$

i.e., conditions (32)-(34) hold. Thus, by Corollary 2.3, equation (57) is oscillatory. 
EXAMPLE 2.

Consider the equation

$$
\left(t^{1 / 3}\left(y^{\prime}(t)\right)^{1 / 3}\right)^{\prime}=\left(1+t^{3}\right) x^{1 / 5}(t / 6)+x^{\mu}(3 t), \quad t \geq 1,
$$

with

$$
y(t)=x(t)+\frac{1}{t^{6}} x^{1 / 7}(t / 3)-t x^{5}(t / 3) .
$$

Here

$$
\alpha=1 / 3, \quad \gamma=1 / 5, \quad \beta=1 / 7, \quad \delta=5,
$$

$\mu$ is the ratio of positive odd integers,

$$
\begin{aligned}
& \tau(t)=t / 6, \quad \sigma(t)=t / 3, \quad \omega(t)=3 t, \quad a(t)=t^{1 / 3}, \\
& q(t)=1+t^{3}, \quad c(t)=1, \quad p_{1}(t)=1 / t^{6}, \quad p_{2}(t)=t .
\end{aligned}
$$

Then, it is easy to see that conditions (i)-(iv), (3) and the first part of (37) hold. Letting $p(t)=1$, we see that condition (66) holds. Letting $\eta(t)=2 t / 3$, we see that

$$
\pi(t):=\omega(\eta(t))=2 t>t,
$$

i.e, condition (36) holds. Since

$$
A\left(t, t_{0}\right)=A(t, 1)=\int_{1}^{t} \frac{\mathrm{d} s}{s}=\ln t
$$

we see that

$$
\int_{t_{0}}^{\infty} Q(s) A^{\gamma / \delta}\left(h_{1}(s), t_{0}\right) \mathrm{d} s=\int_{1}^{\infty} \frac{2^{1 / 25}\left(1+s^{3}\right)}{s^{1 / 25}}\left(\ln \frac{s}{2}\right)^{1 / 25} \mathrm{~d} s=\infty
$$

and

$$
\begin{aligned}
\int_{t_{0}}^{\infty}\left(\frac{1}{a(u)} \int_{\eta(u)}^{u} c(s) \mathrm{d} s\right)^{1 / \alpha} \mathrm{d} u & =\int_{1}^{\infty}\left(\frac{1}{u^{1 / 3}} \int_{2 u / 3}^{u} \mathrm{~d} s\right)^{3} \mathrm{~d} u \\
& =\frac{1}{27} \int_{1}^{\infty} u^{2} \mathrm{~d} u=\infty
\end{aligned}
$$

i.e., conditions (55) and (56) hold. Thus, by Corollary 3.3, a solution $x(t)$ of equation (58) is either oscillatory or satisfies

$$
\liminf _{t \rightarrow \infty}|x(t)|=0 .
$$


Remark 1. There is a number of directions for future research that can be based on the results we obtained here. For example, could there be two different delays in the neutral term, that is, could we have

$$
y(t)=x(t)+p_{1}(t) x^{\beta}\left(\sigma_{1}(t)\right)-p_{2}(t) x^{\delta}\left(\sigma_{2}(t)\right)
$$

with

$$
\sigma_{1}(t) \leq t \quad \text { and } \quad \sigma_{2}(t) \leq t ?
$$

Here we asked that

$$
0<\beta<1 \quad \text { and } \quad \delta>1
$$

What if

$$
\beta>1 \quad \text { and } \quad 0<\delta<1,
$$

or any other combination? Another possibility would be to consider the case where

$$
\lim _{t \rightarrow \infty} A\left(t, t_{0}\right)=\lim _{t \rightarrow \infty} \int_{t_{0}}^{t} a^{-1 / \alpha}(s) \mathrm{d} s<\infty .
$$

It would be of interest also to extend the results here, or for the modifications mentioned above, to higher-order equations such as

$$
\left(a(t)\left(y^{(n-1)}(t)\right)^{\alpha}\right)^{\prime}+q(t) x^{\gamma}(\tau(t))+c(t) x^{\mu}(\omega(t))=0
$$

and

$$
\left(a(t)\left(y^{(n-1)}(t)\right)^{\alpha}\right)^{\prime}=q(t) x^{\gamma}(\tau(t))+c(t) x^{\mu}(\omega(t)),
$$

where $n \geq 3$, and the functions $a, c, q$, and $y$ are as in this paper.

\section{REFERENCES}

[1] AGARWAL, RP.- BOHNER, M.- LI, T.- ZHANG, C.: Oscillation of second-order differential equations with a sublinear neutral term, Carpathian J. Math. 30 (2014), 1-6.

[2] AGARWAL, RP.- GRACE, SR.-O'REGAN, D.: Oscillation Theory for Difference and Functional Differential Equations. Kluwer Academic Publishers, Dordrecht, 2010.

[3] AGARWAL, RP.-GRACE, SR.-O'REGAN, D.: Oscillation Theory for Second Order Linear, Half-Linear, Superlinear and Sublinear Dynamic Equations. Kluwer Academic Publishers, Dordrecht, 2010.

[4] BOHNER, M.-GRACE, SR.-JADLOVSKÁ, I.: Oscillation criteria for second-order neutral delay differential equations, Electron. J. Qual. Theory Differ. Equ. 2017 (2017), no. 60, 1-12. 


\section{OSCILLATORY BEHAVIOUR OF SECOND-ORDER DIFFERENTIAL EQUATIONS}

[5] GRACE, S.R.: Oscillatory behavior of second-order nonlinear differential equations with a nonpositive neutral term, Mediterr. J. Math. 14 (2017), Paper no. 229, 1-12.

[6] GRACE, S. R.-GRAEF, J. R.: Oscillatory behavior of second order nonlinear differential equations with a sublinear neutral term, Math. Model. Anal. 23 (2018), 217-226.

[7] GRAEF, J. R.-GRACE, S. R.-TUNÇ, E.: Oscillatory behavior of even-order nonlinear differential equations with a sublinear neutral term, Opuscula Math. 39 (2019), 39-47.

[8] GRAEF, J.R.-GRACE, S.R.-TUNÇ, E.: Oscillation of even-order nonlinear differential equations with sublinear and superlinear neutral terms, Publ. Math. Debrecen (to appear).

[9] GRAEF, J.R.-GRAMMATIKOPOULOS, M.K.-SPIKES, P. W.: On the asymptotic behavior of solutions of a second order nonlinear neutral delay differential equation, J. Math. Anal. Appl. 156 (1991), 23-39.

[10] GRAEF, J. R.-SPIKES, P. W.: On the oscillation of an nth-order nonlinear neutral delay differential equation, J. Comput. Appl. Math. 41 (1992), 35-40.

[11] HALE, J. K.: Theory of Functional Differential Equations. Springer, New York, 1977.

[12] HARDY, G. H.-LitTlEWOOD, I. E.-POLYA, G.: Inequalities. Reprint of the 1952 edition, Cambridge University Press, Cambridge, 1988.

[13] KOPLATADZE, R.G.-CHANTURIYA, T. A.: Oscillating and monotone solutions of first-order differential equations with deviating argument, Differ. Uravn. 18 (1982), 1463-1465. (In Russian)

[14] LADAS, G.-STAVROULAKIS, I. P.: Oscillation caused by several retarded and advanced arguments, J. Differ. Equations 44 (1982), 134-152.

[15] LI, T.-ROGOVCHENKO, YU.V.: Oscillation of second-order neutral differential equations, Math. Nachr. 288 (2015), 1150-1162.

[16] LI, T.-ROGOVCHENKO, YU. V.-ZHANG, C.: Oscillation results for second-order nonlinear neutral differential equations, Adv. Differ. Equ. 2013 (2013), Paper no. 336, $13 \mathrm{pp}$.

[17] PHILOS, CH. G.: On the existence of nonoscillatory solutions tending to zero at $\infty$ for differential equations with positive delays, Arch. Math. (Basel) 36 (1981), 168-178.

[18] QIN, H.-SHANG, N.-LU, Y.: A note on oscillation criteria of second order nonlinear neutral delay differential equations, Comput. Math. Appl. 56 (2008), 2987-2992.

[19] TAMILVANAN, S.-THANDAPANI, E.-DŽURINA, J.: Oscillation of second order nonlinear differential equation with sub-linear neutral term, Differ. Equ. Appl. 9 (2017), 29-35.

[20] XU, R.-MENG, F.: Some new oscillation criteria for second order quasi-linear neutral delay differential equations, Appl. Math. Comput. 182 (2006), 797-803.

[21] WONG, J.S. W.: Necessary and sufficient conditions for oscillation of second order neutral differential equations, J. Math. Anal. Appl. 252 (2000), 342-352. 


\section{SAID R. GRACE-JOHN R. GRAEF-TONGXING LI —ERCAN TUNÇ}

[22] WU, H.-ERBE, L.-PETERSON, A.: Oscillation of solution to second-order half-linear delay dynamic equations on time scales, Electron. J. Differential Equations 2016 (2016), no. $71,1-15$.

Received September 11, 2020

Said R. Grace

Department of Engineering Mathematics

Cairo University

Giza 12221 Orman

Egypt

E-mail: saidgrace@yahoo.com

John R. Graef

Department of Mathematics

University of Tennessee at Chattanooga

Chattanooga, TN 37403

USA

E-mail: John-Graef@utc.edu

Tongxing $\mathrm{Li}$

School of Control Science and Engineering

Shandong University

Jinan, Shandong 250061

CHINA

E-mail: litongx2007@163.com

Ercan Tunç

Department of Mathematics

Gaziosmanpasa University

60240, Tokat

TURKEY

E-mail: ercantunc72@yahoo.com 